\title{
Auditing National HIV Guidelines and Policies: The United Kingdom CD4 Surveillance Scheme
}

\author{
Alison E. Brown*, Meaghan M. Kall, Ruth D. Smith, Zheng Yin, Alan Hunter and Valerie C. Delpech
}

Health Protection Agency, Colindale, 61 Colindale Avenue, London, NW9 5EQ, UK

\begin{abstract}
The United Kingdom's CD4 surveillance scheme monitors CD4 cell counts among HIV patients and is a national resource for HIV surveillance. It has driven public health policy and allowed auditing of national HIV testing, treatment and care guidelines.

We demonstrate its utility through four example outputs: median CD4 count at HIV diagnosis; late HIV diagnosis and short-term mortality; the timing of first CD4 count to indicate entry into HIV care; and the proportion of patients with CD4 counts $<350$ cells $/ \mathrm{mm}^{3}$ receiving anti-retroviral therapy (ARV).

In $2009,95 \%(61,502 / 64,420)$ of adults living with diagnosed HIV infection had CD4 counts available. The median CD4 count at diagnosis increased from 276 to 335 cells $/ \mathrm{mm}^{3}$ between 2000 and 2009, indicating modest improvements in HIV testing. In $2009,52 \%$ of patients were diagnosed at a late stage of HIV infection $\left(\mathrm{CD} 4<350 \mathrm{cells} / \mathrm{mm}^{3}\right)$; these individuals had a ten-fold risk of dying within a year of their diagnosis compared to those diagnosed promptly. In 2008, the national target of performing a CD4 count within 14 days of diagnosis was met for $61 \%$ of patients. National treatment guidelines have largely been met with $83 \%$ patients with $\mathrm{CD} 4<350$ cells $/ \mathrm{mm}^{3}$ receiving ARV.

The monitoring of CD4 counts is critical to HIV surveillance in the United Kingdom enabling the close monitoring of efforts to reduce morbidity and mortality associated with late diagnosis and underpins the auditing of policies and guidelines. These routine surveillance outputs can be generated at national and local levels to drive and monitor public health policy and prevention efforts.
\end{abstract}

Keywords: Surveillance, CD4 counts, patient outcomes, United Kingdom.

\section{INTRODUCTION}

The monitoring of CD4+ T-lymphocyte cells (CD4 cells) is a central component of the clinical care provided to people living with a diagnosed HIV infection. Without antiretroviral therapy (ARV), CD4 counts gradually decline over the course of an HIV infection [1]. Consequently, CD4 counts per $\mathrm{mm}^{3}$ of the blood provide an indication of patients' levels of immunosuppression. Current British HIV Association (BHIVA) guidelines recommend a patient receive a CD4 count within two weeks of HIV diagnosis [2], and thereafter four times a year to assess disease progression, the requirement for ARV [3], and to monitor the effectiveness of ARV.

The routine monitoring of CD4 counts among HIV patients has created a national resource for HIV surveillance. The availability of longitudinal CD4 counts mapped to individual patient profiles has allowed population trends in immunological status to be monitored. The CD4 surveillance scheme has monitored immunosuppression among adults living with a diagnosed HIV infection in England, Wales and Northern Ireland (E, W and NI) since 1995 [4].

We describe the role CD4 surveillance scheme has had in monitoring the United Kingdom (UK) HIV epidemic and

*Address correspondence to this author at the Health Protection Agency, Colindale, 61 Colindale Avenue, London, NW9 5EQ, UK; Tel: 020 83277559; E-mail: Alison.brown@hpa.org.uk demonstrate how the data can be linked to other surveillance programmes in creative ways to inform public health priorities and to audit national guidelines. We provide four examples of the utility of CD4 surveillance: 1) the median CD4 count at HIV diagnosis; 2) late and very late HIV diagnosis and associated short-term mortality; 3 ) the timing of first CD4 count as a proxy of entry into HIV care; 4) the proportion of patients with a CD4 count $<350$ cells $/ \mathrm{mm}^{3}$ receiving ARV.

\section{METHODS}

\section{The CD4 Surveillance Scheme}

All laboratories performing CD4 cell counts in E, W and NI that are registered with the National External Quality Assessment Scheme for Leukocyte Immunophenotyping (UK NEQAS) are invited to participate in the CD4 surveillance scheme [5]. Data are currently reported voluntarily from over 60 laboratories, representing approximately $80 \%$ of laboratories that process $\mathrm{CD} 4$ counts.

Data collected include limited patient identifiers, date of CD4 test and CD4 counts. The limited identifiers enable reports from patients who have had multiple CD4 counts undertaken (including in different laboratories) to be linked to the same patient both within the CD4 surveillance scheme and to other surveillance systems.

\section{Linkage to Other HIV Surveillance Systems}

Data from the CD4 surveillance scheme are linked routinely via limited patient identifiers to the Survey of 
Prevalent HIV Infections Diagnosed (SOPHID) $[6,7]$ and the survey of new HIV diagnoses, AIDS and deaths [8]. SOPHID is an annual census of adults ( $\geq 15$ years) accessing HIV treatment sites in E, W and NI. Demographic, exposure and clinical information are also collected. The new HIV diagnoses database collates the number of new HIV diagnoses, first AIDS diagnoses and deaths in E, W and NI reported by clinicians and laboratories, including exposure and demographic data.

Scottish data from the CD4 surveillance scheme, and reports of new HIV diagnoses are provided by Health Protection Scotland [9] and incorporated to create a UK dataset. HIV diagnoses made during pregnancy, and pregnancy events among diagnosed HIV positive women are reported by the National Study of HIV in Pregnancy and Childhood and linked to SOPHID and the new HIV diagnosis database.

\section{Study Population}

Data from the CD4 surveillance scheme, linked to SOPHID and new diagnoses database are used throughout. Unless otherwise specified, data are for the UK and cover the years 2000 to 2009. Data are presented by HIV exposure group, sex, ethnicity, country of birth and age-group. Adults are defined as being aged 15 years or over. Data with incomplete $\mathrm{CD} 4, \mathrm{ARV}$ and/or relevant demographic fields (e.g. HIV infection route) are excluded from the analysis; in 2009 , missing data accounted for $5 \%,(2,918 / 64,420), 1 \%$ (714) and $3 \%,(2,209)$ respectively.

\section{Median CD4 Count at HIV Diagnosis}

The median CD4 at diagnosis (where CD4 counts are taken within 91 days of diagnosis) provides an indication of the level of immunosuppression at diagnosis [1]. Patients without a CD4 count are excluded from this analysis.

\section{Late and Very Late HIV Diagnosis and Associated Mortality}

Patients who are diagnosed with a CD4 count $<350$ cells $/ \mathrm{mm}^{3}$ (within 91 days of HIV diagnosis) are defined to have been diagnosed at a late stage of HIV infection; those diagnosed with a CD4 count $<200$ cells $/ \mathrm{mm}^{3}$ as having a very late stage HIV infection. Linkage to death reports from the Office for National Statistics [10] and clinician reports enables the monitoring of short term mortality (deaths within one year of HIV diagnosis) by late and prompt (CD4 $>350$ cells $/ \mathrm{mm}^{3}$ ) HIV diagnosis.

\section{Time from Diagnosis to First CD4 Count}

BHIVA guidelines suggest that all patients should have their first CD4 count measured within two weeks of HIV diagnosis [2]. The length of time between HIV diagnosis and first CD4 count is used as a proxy for the proportion of patients incorporated in to HIV care following diagnosis. Patients who did not have a CD4 count within a year of diagnosis were excluded from the analysis.

\section{Patients with a CD4 Count $<350 \mathrm{Cells} / \mathrm{mm}^{3}$ who Receive ARV}

BHIVA guidelines recommend that all patients should begin ARV when their CD4 cell counts reach $<350$ cells $/ \mathrm{mm}^{3}$. We monitor the proportion of patients with CD4 $<350$ cells $/ \mathrm{mm}^{3}$ receiving ARV.

\section{Statistical Methods}

Descriptive epidemiology is the focus of the paper, but hypothesis tests have been used to supplement the data where appropriate.

\section{RESULTS}

\section{Coverage}

The CD4 surveillance scheme has a cumulative total of 1,726,736 CD4 cell counts from 127,299 HIV-infected adults in the UK up to the end of 2009. Eighty per cent $(54,218 / 67,557)$ of patients diagnosed between 2000 and 2009 had a CD4 count available within 91 days of HIV diagnosis. In 2009 alone, $84 \%(5,525 / 6,556)$ of adults newly diagnosed with HIV had a CD4 count within 91 days of diagnosis. In 2009, 91\% (58,407/64,359) of adults living with diagnosed HIV infection in the UK (SOPHID) were linked to the CD4 surveillance scheme, and $62 \%$ $(40,013 / 64,359)$ had CD4 counts available during 2009. After linking datasets, overall, $84 \%(5,525 / 6,556)$ of adults newly diagnosed with HIV had a CD4 count within 91 days of diagnosis, and $95 \%(61,502 / 64,420)$ of adults accessing care had a CD4 count available in 2009.

\section{Median CD4 Count at HIV Diagnosis}

Overall, the median CD4 count at diagnosis has gradually increased by $21 \%$ from 276 in 2000 to 335 in 2009 (Fig. 1). The largest increase in median CD4 count between was observed among heterosexual men (59\% increase), followed by non-pregnant women $(21 \%)$ and men who have sex with men (MSM, 18\%). The median CD4 count among pregnant women has remained relatively stable over the last decade (353 in 2000 and 346 in 2009).

In 2009, the highest median CD4 count at HIV diagnosis was among MSM (411) and lowest among heterosexual men (254). Since 2000, the median CD4 count at HIV diagnosis of women detected through antenatal screening has been consistently higher than among other women; the figures in 2009 were 345 and 279 respectively.

\section{Late HIV Diagnoses}

In 2009 , an estimated $52 \%(2,867 / 5,525)$ of adults were diagnosed late (Fig. 2) and $30 \%(1,656 / 5,525)$ were diagnosed very late. The lowest percentage of adults diagnosed late was among MSM $(39 \% ; 878 / 2,227)$ and highest among heterosexual men $(66 \% ; 712 / 1,071)$. Fiftynine per cent $(969 / 1,644)$ of heterosexual women were diagnosed late and 52\% (62/120) of injecting drug users.

Among heterosexual men and women, those born outside of the UK were more likely to be diagnosed late compared to those born in the UK: $64 \%(1,203 / 1,876)$ and $52 \%(252 / 488)$ respectively. By region of birth and exposure group, the highest percentage of people diagnosed late was among heterosexuals born in Africa $(67 \%, 1,043 / 1,560)$. There was also variation between age groups, with two-thirds $(67 \%$, 477/716) of older adults (aged 50 and above) being diagnosed late, compared with half $(50 \%, 2,390 / 4,809)$ of younger adults. 


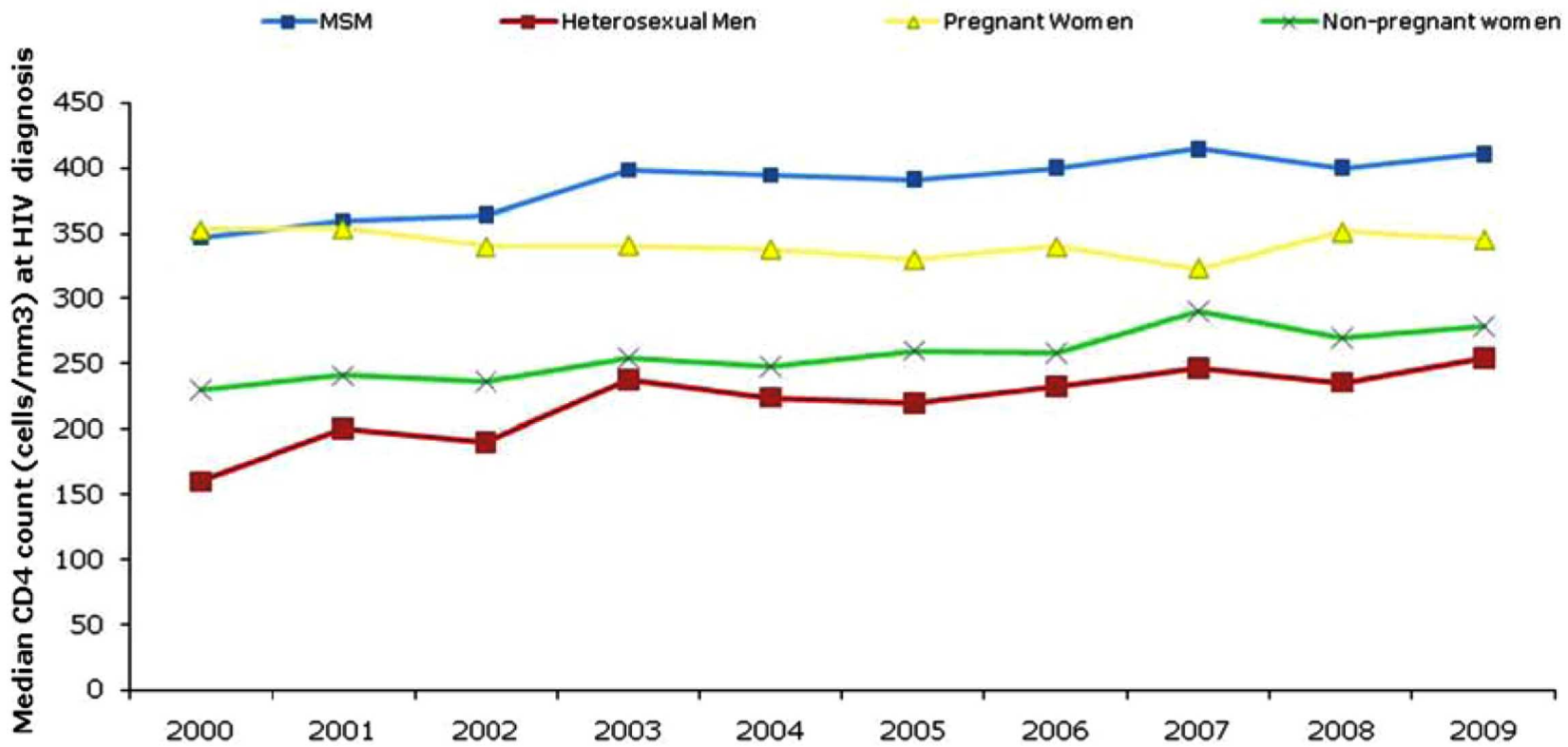

Fig. (1). The median CD4 count at HIV diagnosis by exposure group, United Kingdom: 2000-2009.

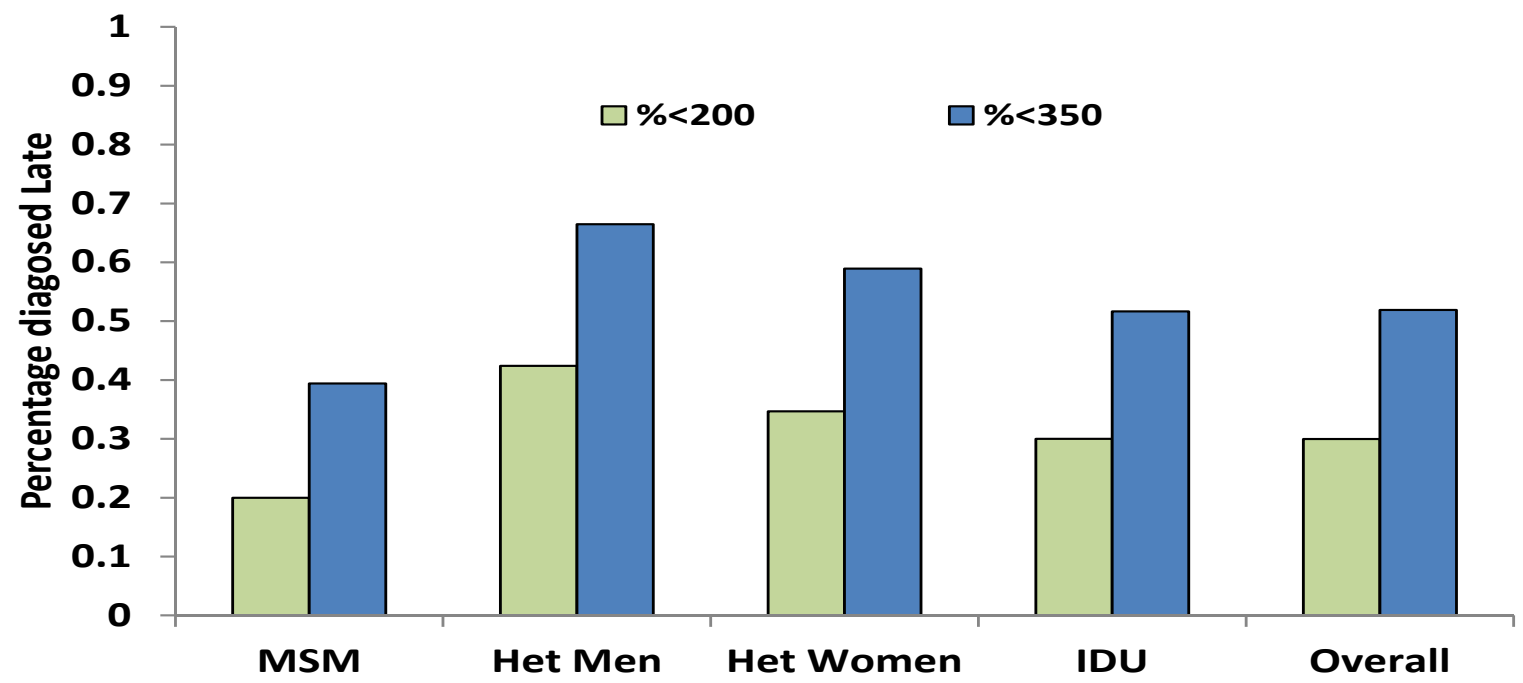

\section{* CD4 count within 91 days of HIV diagnosis}

Fig. (2). Percentage of adult diagnosed late $(C D 4<350)$ and very late $(C D 4<200)$ by exposure group, United Kingdom: 2009.

\section{Short-Term Mortality}

In $2009,91 \%(116 / 128)$ of deaths within one year of diagnosis occurred among individuals diagnosed late. Late diagnosis increased the risk of short-term mortality ten-fold, with $4.1 \%(107 / 2,599)$ of those diagnosed late dying within 12 months of diagnosis, compared to $0.3 \%(8 / 2,402)$ of those diagnosed promptly $(\mathrm{p}<0.001)$ (Fig. 3). By risk group, death within one year of diagnosis was $2.9 \%$ in MSM, $6.2 \%$ in heterosexual men, and $2.2 \%$ in heterosexual women. This compares to $0.2 \%, 0.6 \%$, and $0.3 \%$ among those diagnosed promptly, respectively.

\section{Time from Diagnosis to First CD4 Count}

In $\mathrm{E}, \mathrm{W}$ and NI, 5,909 adults were diagnosed with HIV infection in 2008. Of these, $61 \%(2,975 / 4,868)$ had their first CD4 count within 14 days of their diagnosis (this excludes patients who did not have a CD4 count reported within 12 months of HIV diagnosis). Seventy-seven per cent $(3,752)$ of patients had their first CD4 count within one month, and $92 \%(4,477)$ within three months of diagnosis. $(88 \%)$ "of those who survived" were seen for care in 2009 , 142 had died by 2009 and 5,080 (88\%) were seen for care in 2009. There was no significant difference between patient exposure to HIV, ethnicity or age group (data not shown). 


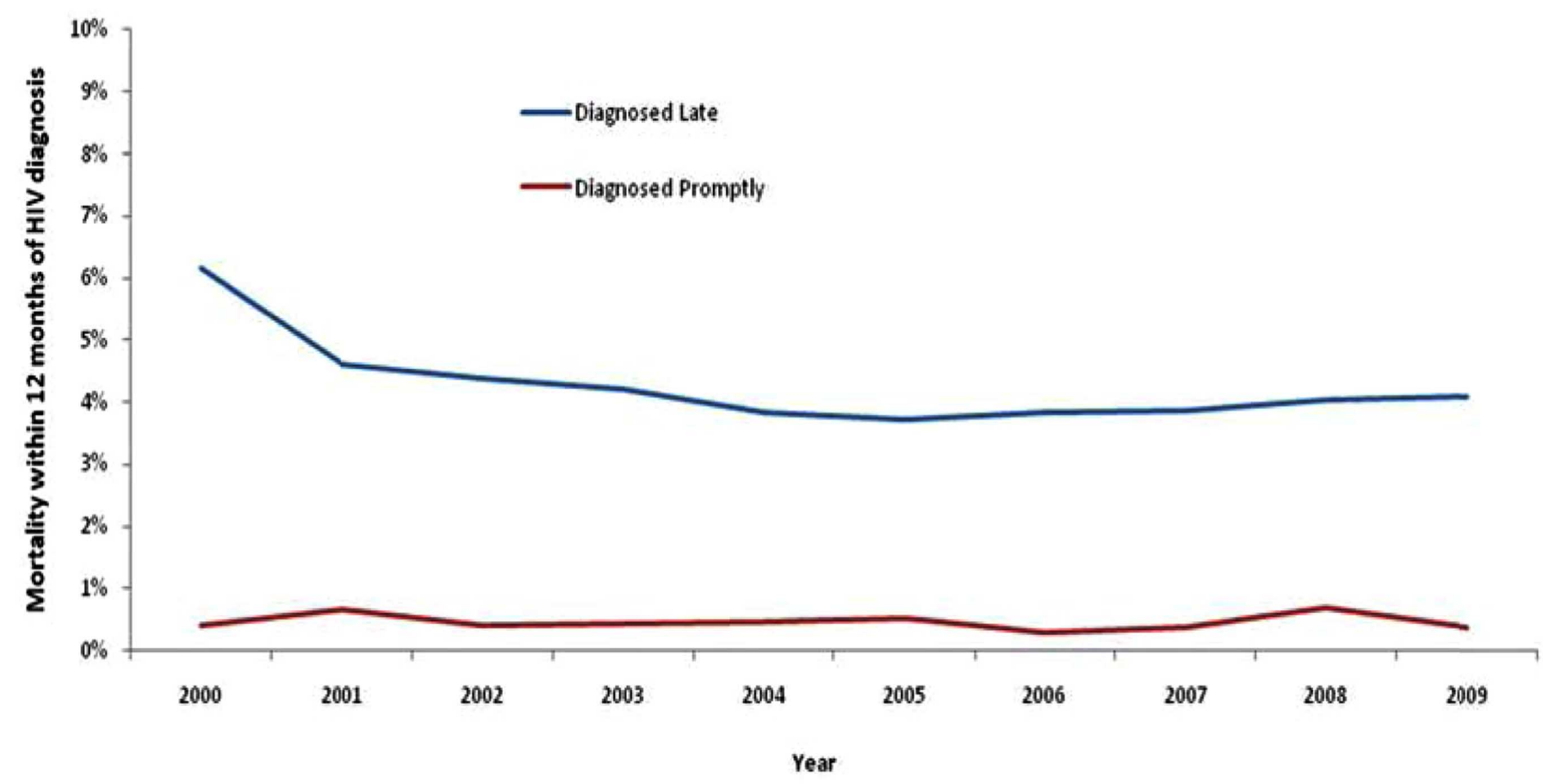

Fig. (3). Mortality within one year of HIV diagnosis among adults diagnosed late (CD4<350 cells) and promptly (CD4 $>350$ cells), United Kingdom: 2009.

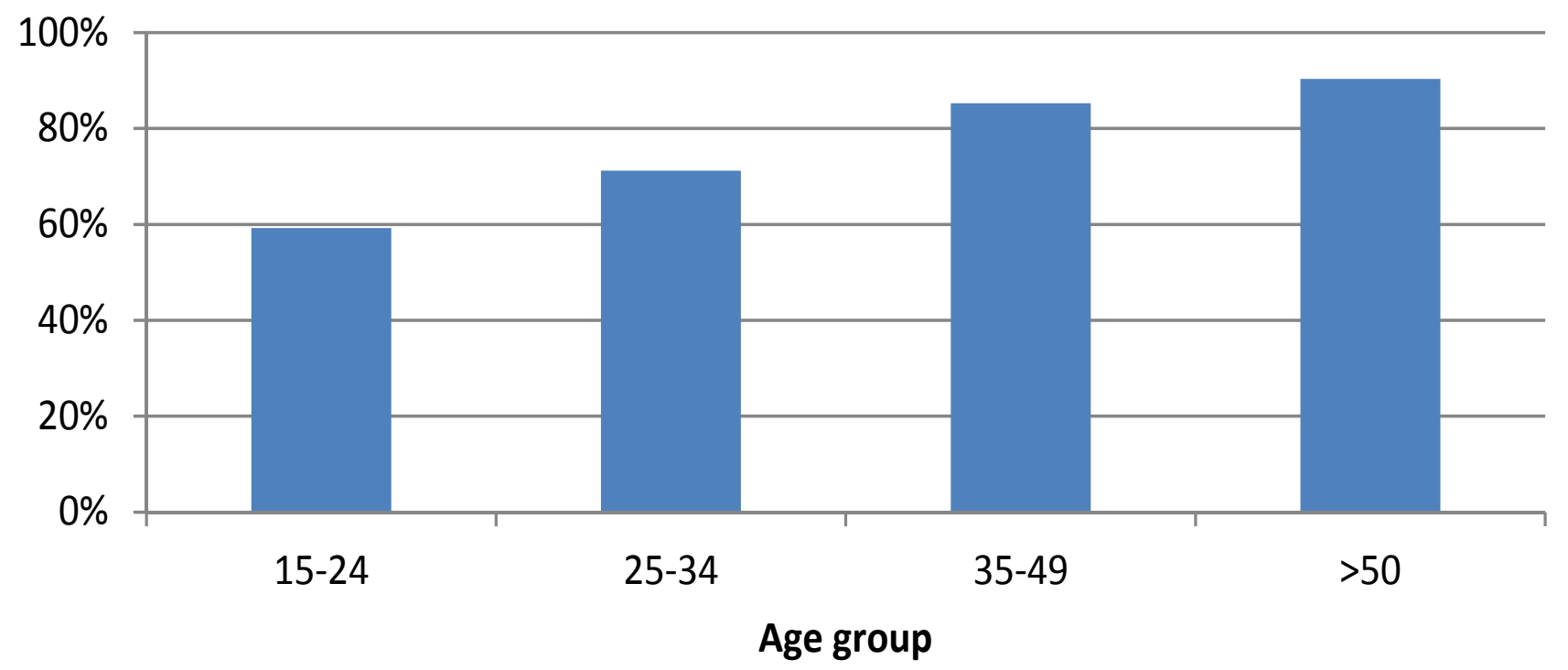

Fig. (4). Proportion of HIV pateints with CD4 counts <350 cells receiving ARV, UK: 2009.

\section{Percentage of Patients with a CD4 Count $<350$ Cells $/ \mathrm{mm}^{3}$ who Receive ARV}

The percentage of HIV patients with CD4 counts $<350$ cells $/ \mathrm{mm}^{3}$ receiving ARV remained stable at about $74 \%$ between $2000-2007$, increasing to $81 \%(14,243 / 17,653)$ in 2008 and $83 \%(14,240 / 17,257)$ in 2009 . Of the 3017 patients with CD4 counts $<350$ not receiving ARV 2009, 24\% (709) were diagnosed with HIV in 2009 (Fig. 4).

In 2009, 81\% (5,117/6,309) of MSM and 82\% (446/545) of injection drug users (IDUs) with CD4 counts $<350$ cells $/ \mathrm{mm}^{3}$ were receiving ARV. Equivalent figures among heterosexual men and women were $88 \%(3,486 / 3,977)$ and $81 \%(4,484 / 5,516)$ respectively $(\mathrm{p}=0.400)$. By ethnic group, the percentage with a CD4 count $<350$ cells $/ \mathrm{mm}^{3}$ receiving ARV was $82 \%(5,668 / 6,954)$ among whites, $81 \%(342 / 422)$ among black Caribbean and $85 \%(5,549 / 6,501)$ among black Africans $(p=0.200)$. In the same year, the percentage of patients with CD4 counts $<350$ cells $/ \mathrm{mm}^{3}$ receiving therapy increased with age (Fig. 4); 59\% (340/574) among those aged $15-24$ years and $90 \%(3,201 / 3,543)$ among those aged 50 years and over $(\mathrm{p}<0.001)$. 


\section{DISCUSSION}

The routine reporting of $\mathrm{CD} 4$ counts at diagnosis and subsequent follow-up care forms an essential component of the surveillance of HIV in the UK. These data linked to new diagnoses and annual surveys of persons in HIV care provide a detailed understanding of the HIV epidemiology in the UK and enable the auditing of national HIV testing, treatment and care guidelines. These routine surveillance outputs can be generated at the national and local level to drive and monitor public health policy and prevention efforts.

In the UK, the completeness of CD4 data is high: over $95 \%$ of people living with diagnosed HIV infection had CD4 data available in 2009. Our findings show that over half of newly diagnosed patients had already reached a stage in their infection where treatment should have begun; such patients have a ten-fold risk of mortality within a year compared to those diagnosed promptly. Six out of ten patients newly diagnosed have a CD4 count within 14 days of HIV diagnosis; the best practise target [2] and nine out of ten had a count within 91 days of diagnosis and were seen for care within the next twelve months. Four in five of patients with CD4 counts $<350$ cells $/ \mathrm{mm}^{3}$ are received treatment, and therefore national guidelines [3] are largely being met

Routinely monitoring late diagnosis is important to evaluate HIV testing policies and programmes at the national level. High rates of late diagnoses reflect the proportion of infected individuals who remain undiagnosed, which in the UK is estimated at around 22,200 , or $26 \%$ of all people living with HIV [11]. Individuals unaware of their HIV status remain infectious for many years, increasing the risk of onward transmission to sexual partners. Prompt HIV diagnosis prevents further HIV transmission through ensuring patients' viral load is low (through regular monitoring and/or administering anti-retroviral therapy where clinically appropriate) and providing earlier opportunities for partner notification and behaviour change counselling.

The percentage of late diagnoses were especially high among heterosexual men $(66 \%)$ followed by heterosexual women (59\%) and lower among MSM (39\%). Over the past decade, an increase in the median CD4 count at diagnosis across risk groups reflects modest improvements in HIV testing. The higher median CD4 count among women diagnosed during pregnancy compared to non-pregnant women reflects the success of the antenatal screening programme. However, in 2009 only MSM had a median CD4 count at diagnosis over 350 cells $/ \mathrm{mm}^{3}$ indicating continued efforts are needed to test and diagnose people living with HIV, particularly black African communities in the UK, at an earlier stage of their infection.

CD4 surveillance, taken with surveillance of deaths in HIV-infected individuals, also makes it possible to quantify the burden of mortality attributable to late diagnosis. Nine in ten deaths occurring within one year of HIV diagnosis are among those diagnosed late; many of these deaths could have been prevented through prompt diagnosis. In addition to the impact on short-term mortality, late diagnoses also affect morbidity. In studies of untreated HIV infection, risk of developing an AIDS-defining illness is greatly increased at lower CD4 counts, with particularly high risk in those with a CD4 count less than 200 cells $/ \mathrm{mm}^{3}$ [12]. Lower CD4 counts have also been associated with increased risk of nonAIDS related illnesses including cardiovascular, renal, and hepatic disease and some malignancies $[13,14]$. Even after starting ARV, individuals who start late experience suboptimal benefit from ARV compared to those who start early, due to their longer exposure to HIV-associated inflammation and immune activation [15-17].

The high rates of late HIV diagnoses mean that establishing rapid pathways from HIV diagnosis into care is essential. Over a third of patients newly diagnosed do not have a CD4 count within 14 days of HIV diagnosis; the best practise target [2]. Furthermore, it is good clinical practice to request $\mathrm{CD} 4$ testing at the time of HIV diagnosis. Consequently, CD4 counts processed at HIV diagnosis may not necessarily reflect patients accessing subsequent care. If patients who have CD4 counts taken within four days of their HIV diagnosis are excluded, $(52 \%, 1560)$, this provides a minimum estimate of $29 \%$ receiving CD4 counts within 14 days of HIV diagnosis. Nevertheless, it is likely that the majority of patients who have a CD4 count processed at HIV diagnosis are also assimilated into care rapidly; nine in ten of patients diagnosed in 2008 were seen for care in 2009. Factors associated with being lost to follow up are including younger age; being recently diagnosed; and black African ethnicity [18].

It is encouraging that four in five patients with CD4 counts $<350$ cells $/ \mathrm{mm}^{3}$ are receiving ARV, demonstrating providers are adhering to national guidelines [3]. The increase from 2008 reflects the change in BHIVA guidelines [3] (previous guidelines recommended ARV commence when CD4 counts $<200$ cells $/ \mathrm{mm}^{3}$ ). One quarter of immunosuppressed patients not receiving ARV were diagnosed with HIV within the past 12 months; these individuals may not yet have had the opportunity to start ARV. Importantly, patient ethnicity and exposure to HIV are not associated with not receiving ARV. However, age is important with young people with CD4 counts $<350$ cells less likely to receive ARV. Younger people find adherence to ARV more difficult [19] or may have been infected for a shorter length of time and therefore have a different perception of the benefits of ARV compared to those who have experienced symptoms [20]. The reasons for poorer ARV uptake among young people in need of treatment require further exploration.

\section{Implications for Policy}

The monitoring of late HIV diagnoses has been routinely measured nationally and at the local level for many years. The late diagnosis indicator has already been implemented in London as part of the London Tool Kit for commissioners. It has been valuable in driving and tracking public health efforts to expand HIV testing in the capital.

The expansion of HIV testing in high prevalence areas has been recommended through BHIVA guidelines [21] as well as in the National Institute for Health and Clinical Excellence (NICE) guidance [22]. The guidance advocates the development of local policies for HIV testing among black Africans and MSM and the routine offer of a test in a range of health care settings in high prevalence areas. The 
continued monitoring of late HIV diagnoses will evaluate the success of these policies.

The expansion of HIV testing in to non-traditional settings intensifies the need for continuing auditing of rapid pathways into care. This pathway will facilitate prompt treatment for those diagnosed at a late stage of infection. In order to ensure patients diagnosed with HIV receive high quality care and treatment, the London HIV consortium has developed four outcome measures. In addition to monitoring prompt access to care following diagnosis, this suite of outcomes also measures ARV adherence, ARV success in achieving undetectable viral loads, the prevention of patients in care reaching levels of severe immunosuppression and mortality rates. The measures have been successfully implemented in London and will be rolled-out nationally; the CD4 surveillance scheme is fundamental to its success.

\section{Limitations}

The limitations associated with CD4 surveillance are as follows. Patients who have CD4 counts linked to their patient records will not be representative of patients with sub-optimal attendance to care, who are more likely to be vulnerable and experience poorer health outcomes. Patients newly diagnosed are also less likely to have CD4 counts available. However, the overall linkage between HIV surveillance systems is very high at $95 \%$, minimising this bias.

\section{CONCLUSION}

The health care costs of HIV are substantial; over $£ 600$ million is spent each year to provide health care and treatment of the 65,300 people living with a diagnosed HIV infection. National HIV surveillance systems must be responsive to the changes in the epidemic and advances in treatment and care. The monitoring of CD4 counts is a critical core function of HIV surveillance in the UK enabling the close monitoring of efforts to reduce morbidity and mortality associated with late diagnosis and underpins the auditing of national policies and guidelines. It is important the national surveillance systems such as the CD4 surveillance scheme continue to be evaluated to ensure their efficiency and effectiveness [23].

\section{AUTHOR CONTRIBUTION}

1. Alison Brown: Principal HIV scientist within the HIV and STI Department at the Health Protection Agency, lead in the design and writing of paper and responsible for final manuscript.

2. Meaghan Kall: Scientist within the HIV and STI Department at the Health Protection Agency helped conduct analyses, provided comments and advice on drafts of the paper.

3. Ruth Smith: Lead scientist within the HIV and STI Department at the Centre for Infections, helped conduct analyses, provided comments and advice on drafts of the paper.

4. Zheng Yin: Scientist within the HIV and STI Department at the Health Protection Agency helped conduct analyses, provided comments and advice on drafts of the paper.
5. Alan Hunter: Information Analyst for the CD4 surveillance scheme. HIV and STI Department at the Health Protection Agency helped conduct analyses, provided comments and advice on drafts of the paper.

6. Valerie Delpech: Consultant epidemiologist and lead for HIV surveillance within the HIV and STI department at the Health Protection Agency, contributed substantially to the study design and writing of the paper.

\section{ACKNOWLEDGEMENTS}

The authors would like to acknowledge the continuing collaboration of those who contribute to HIV surveillance in the UK. We also thank Health Protection Scotland and the National Study of HIV in Pregnancy and Childhood, Institute of Child Health, University College London for providing Scottish surveillance data and pregnancy data respectively.

\section{FUNDING}

CD4 Surveillance scheme: Funded by the Department of Health.

National SOPHID survey: Funded by the Department of Health.

London SOPHID survey: Funded by the NHS London Specialised Commissioning Group.

HIV and AIDS New Diagnoses: Funded by the Department of Health.

\section{CONFLICT OF INTEREST}

No paper resembling the enclosed article has been or will be published elsewhere.

\section{REFERENCES}

[1] Pantaleo, G, Graziosi C, Fauci AS. New concepts in the immunopathogenesis of human immunodeficiency virus infection. N Engl J Med 1993; 328(5): 327-35.

[2] British HIV Association. Standards for HIV Clinical care. 2007 Available at: http:/www.bhiva.org/documents/Guidelines/Standard s/StandardsHIVClinicalCare.pdf [Accessed 14 April 2011].

[3] Gazzard, BG. British HIV Association Guidelines for the treatment of HIV-1-infected adults with antiretroviral therapy 2008. HIV Med 2008; 9(8): 563-608.

[4] Health Protection Agency. The CD4 Surveillance Scheme. Available at: http://www.hpa.org.uk/web/HPAweb\&Page\&HPAwe bAutoListName/Page/1201094588994 [Accessed 14 April 2011].

[5] Chadborn TR, Delpech VC, Sabin CA, Sinka K, Evans BG. The late diagnosis and consequent short-term mortality of HIV-infected heterosexuals [England and Wales, 2000-2004]. AIDS 2006; 20(18): 2371-9.

[6] Rice, BD, Payne LJ, Sinka K, Patel B, Evans BG, Delpech V. The changing epidemiology of prevalent diagnosed HIV infections in England, Wales, and Northern Ireland, 1997 to 2003. Sex Transm Infect 2005; 81(3): 223-9.

[7] McHenry A, Macdonald N. Sinka K, Mortimer J, Evans B. National assessment of prevalent diagnosed HIV infections. Commun Dis Public Health 2000; 3(4): 277-81.

[8] Smith RD, Delpech VC, Brown AE, Rice BD. HIV transmission and high rates of late diagnoses among adults aged 50 years and over. AIDS 2010; 24(13): 2109-15.

[9] Health Protection Scotland. Available at: http://www.hps.scot.nhs. uk/search/atozdetail.aspx?subject $=27$ [Accessed 14 April 2011].

[10] Office of National Statistics. Available at: http://www.statistics. gov.uk/hub/index.html [Accessed 14 April 2011]. 
[11] Health Protection Agency. HIV in the United Kingdom: 2010 report. Available at: http://www.hpa.org.uk/web/HPAwebFile/HPA web_C/ 1287145367237 [Accessed 14 April 2011].

[12] Phillips AN, Lepri AC, Lampe F, Johnson M, Sabin CA. When should antiretroviral therapy be started for HIV infection? Interpreting the evidence from observational studies, AIDS 2003; 17(13): 1863-9.

[13] Lichtenstein KA, Armon C, Buchacz K, et al. Low CD4 $4^{+} \mathrm{T}$ Cell Count Is a Risk Factor for Cardiovascular Disease Events in the HIV Outpatient Study. Clin Infect Dis 2010; 51(40): 435-47.

[14] Phillips AN. Neaton J, Lundgren JD. The role of HIV in serious diseases other than AIDS. AIDS 2008; 22(18): 2409-18.

[15] Deeks SG, Kitchen CM, Liu L, et al. Immune activation set point during early HIV infection predicts subsequent CD4+ T-cell changes independent of viral load. Blood 2004; 104(4): 942-7.

[16] Kelley CF, Kitchen CM, Hunt PW, et al. Incomplete peripheral $\mathrm{CD} 4+$ cell count restoration in HIv-infected patients receiving long-term antiretroviral treatment. Clin Infect Dis 2009; 48(6): 787-94.

[17] Gras L, Kesselring AM, Griffin JT, et al. CD4 cell counts of 800 cells $/ \mathrm{mm}^{3}$ or greater after 7 years of highly active antiretroviral therapy are feasible in most patients starting with 350 cells $/ \mathrm{mm}^{3}$ or greater. J AIDS 2007; 45(2): 183-92.
[18] Rice BD, Delpech VC, Chadborn TR, Elford J. Loss to Follow-Up Among Adults Attending Human Immunodeficiency Virus Services in England, Wales and Northern Ireland. Sex Transm Dis 2011; 38(8): 685-90.

[19] Schönnesson LN, Williams ML, Ross MW, Diamond PM, Keel B. Three types of adherence to HIV antiretroviral therapy and their association with AIDS diagnosis, medication side-effects, beliefs about antiretroviral therapy, and beliefs about HIV disease. Int J STD AIDS 2007; 18(6): 369.

[20] Veinot TC, Flicker SE, Skinner HA, et al. "Supposed to make you better but it doesn't really": HIV-positive youths' perceptions of HIV treatment. J Adolesc Health 2006; 38(3): 261-7.

[21] BHIVA, BASHH and BIS. UK National Guidelines for HIV Testing 2008. Available at: http://www.bhiva.org/documents/Guide lines/Testing/GlinesHIVTest08.pdf [Accessed 14 April 2011].

[22] National Institute for Health and Clinical Excellence, NICE public health guidance 33: Increasing the uptake of HIV testing to reduce undiagnosed infection and prevent transmission among black African communities living in England, 2011. Available at: http://guidance.nice.org.uk/PH33 [Accessed 14 April 2011].

[23] Centers for Disease Control and Prevention. Updated guidelines for evaluating public health surveillance systems: recommendations from the guidelines working group. MMWR 2001; 50(No. RR-13): $1-35$.

(C) Brown et al:; Licensee Bentham Open.

This is an open access article licensed under the terms of the Creative Commons Attribution Non-Commercial License (http:/creativecommons.org/licenses/by-nc/ 3.0/) which permits unrestricted, non-commercial use, distribution and reproduction in any medium, provided the work is properly cited. 